CAHIERS DE

NARRATOLOGIE

\section{Cahiers de Narratologie}

Analyse et théorie narratives

32 | 2017

Récit et argumentation, interactions, lieux et dispositifs sociaux

\title{
Récit et argumentation en français : cercle vicieux ou vertueux?
}

Nicole Biagioli

\section{CpenEdition}

Journals

Electronic version

URL: http://journals.openedition.org/narratologie/7819

DOI: 10.4000/narratologie.7819

ISSN: 1765-307X

Publisher

LIRCES

Electronic reference

Nicole Biagioli, «Récit et argumentation en français : cercle vicieux ou vertueux ? », Cahiers de

Narratologie [Online], 32 | 2017, Online since 21 December 2017, connection on 04 May 2019. URL:

http://journals.openedition.org/narratologie/7819; DOI : 10.4000/narratologie.7819

This text was automatically generated on 4 May 2019.

Article L.111-1 du Code de la propriété intellectuelle. 


\title{
Récit et argumentation en français : cercle vicieux ou vertueux?
}

\author{
Nicole Biagioli
}

1 Notre étude se situe dans le champ de la didactique du français langue première et a pour objet le rôle et la place du récit et de l'argumentation dans l'enseignement du français. À «la question de savoir si c'est l'argumentation ou le récit qui est le tout du langage ", Maingueneau oppose la complémentarité du récit qui «ne s'impose pas à l'autre », mais « lui propose au contraire une scénarisation narrative du monde dans laquelle il peut être partie prenante », et de l'argumentation qui « impose à l'autre son mode de raisonnement et ses arguments" (Maingueneau, 1998, 7). Voilà pour il, la non-personne, et le tu, l'allocutaire. Mais en dernier ressort c'est le je, le locuteur, qui décide de l'identification des deux types de discours. Eco $(1985,140)$ le rappelle: «On peut toujours aborder, du point de vue de la construction narrative, des textes qui ne semblent raconter aucune fabula ». D'autre part, l'importance de la liberté interprétative montre à quel point la présentation des énoncés influence l'apprentissage. Le recours aux extraits est en grande partie responsable des risques de confusion entre le discours dominant dans une cellule textuelle et celui qui domine dans le texte. Les «interactions entre récit et argumentation » : premier axe de cadrage du séminaire objet de la présente publication, sont difficiles à détecter dans un texte et à réaliser sur commande, alors qu'elles font partie du discours quotidien.

2 En tant que "lieu de l'argumentation narrative ", deuxième axe du cadrage, l'école entraîne une fracture culturelle pour les élèves dont la sphère langagière privée est très éloignée de la langue qu'elle impose, d'autant que cette langue est perçue comme la clef de l'intégration linguistique des divers groupes sociaux dans la société française (Guérin, 2010). L'explication se trouve en partie dans les «dispositifs, techniques et usages » des apprentissages du récit et de l'argumentation, troisième axe du cadrage. Ceux-ci présentent un quadruple feuilletage de :

3 - supports d'enseignement (textes, images) ;

- échanges entre élèves et enseignants lorsqu'ils se servent du récit et/ou l'argumentation pour communiquer ; 
- échanges entre élèves et enseignants lorsque le récit et/ou l'argumentation constituent l'objet didactique étudié ;

- genres scolaires utilisés pour évaluer les connaissances et les compétences.

4 L'énumération atteste la complexité des situations de communication vécues en classe et la diversité des usages qui s'y reflètent.

5 Ce qui invite à se pencher sur le quatrième axe du cadrage : la formation. Pour tâcher de répondre aux questions qu'il pose: «Comment est organisée la formation scolaire, universitaire aux techniques argumentatives et/ou aux pratiques narratives? En quel sens narration et argumentation peuvent-elles faire l'objet d'une appropriation culturelle par les (futurs) citoyens ? Comment distinguer et concilier ce ferment de la rationalité occidentale qu'est le bon usage de l'argumentation dans les débats publics, et une approche narrative accessible et maitrisable par un public plus large? Parlera-t-on d'éducation, d'apprentissage ou d'accompagnement vers l'exposition et l'expression de soi dans l'espace public ", nous analyserons successivement l'impact de la forme scolaire sur les apprentissages narratifs et discursifs, l'historique de l'évolution du récit et de l'argumentation dans les pratiques scolaires, leur place dans la progression curriculaire, et dans la refondation de l'école de 2013.

\section{L'impact de la forme scolaire}

6 Le concept de forme scolaire permet de décrire le cadre des apprentissages (Reuter, 2012). Ce cadre est paradoxal : on vit dans l'école, donc on y communique de façon authentique, mais en même temps on y crée des situations artificielles pour apprendre à communiquer mieux ou différemment que dans la vie. La forme scolaire trace une frontière entre l'école et la vie, ce qui ne signifie pas nécessairement une barrière, mais plutôt une porosité qui permet une influence réciproque.

\section{L'emprise idéologique}

7 Nous donnerons deux exemples des interactions entre école et société, l'un pour l'argumentation, l'autre pour le récit. Le renouveau des études sur l'argumentation a entrainé la réintroduction de la rhétorique ancienne dans les programmes de français par réaction au traumatisme de la $2^{\text {nde }}$ guerre mondiale, interprétée comme la conséquence de l'endoctrinement des masses par des discours totalitaires. Nous renvoyons à l'historique qu'en donne Plantin $(1996,10)$. En France, cette refondation a été freinée jusque dans les années 70 par les critiques marxistes et psychanalytiques qui y voyaient une « tentative illusoire du sujet pour se rendre maitre de son discours » (ibid., 11). Puis la jonction de l'étude de l'argumentation et de la toute nouvelle linguistique du discours a eu raison des résistances en fournissant des outils pour étudier « les argumentations dites "quotidiennes" ou "communes", menées hors cadre institutionnel» (ibid., 12). Les conditions étaient réunies pour didactiser l'argumentation. En 1974, le protocole de travail d'un extrait du Contrat social, propose comme thème de débat « Rousseau est-il le père des régimes démocratiques, socialistes ou fascistes ? » et comme thème d'étude « Les hommes politiques contemporains parlent souvent de nouveau Contrat social. Qu'entendent-ils par là? Que représente cette notion pour votre entourage?», ( $\mathrm{La}$ littérature et les idées, Littérature et langages, Nathan, t. 4, 305). 
8 Notre second exemple est moins conjoncturel. Les manuels de l'école primaire de la fin du $\mathrm{XIX}^{\mathrm{e}}$ siècle regorgent de récits moralisateurs. Les manuels modernes en gardent la trace, quoique l'injonction à suivre le modèle y cède la place à la réflexion. Mots et merveilles $5^{e}$ (Magnard, 1982) fait suivre la lecture d'un conte qui oppose le bonheur des pauvres à l'insatisfaction des riches, d'une «causerie -débat: Est-il vrai que l'argent ne fait pas le bonheur? » (ibid., 88).

9 Le récit édifiant rend implicite la leçon qui le sous-tend. L'argumentation canonique poursuit et dénonce l'implicite. Deux modèles d'éducation à l'éthique : persuasion et réflexion entre lesquels l'école hésite en fonction des époques et des publics.

\section{L'emprise culturelle}

10 Si évidente qu'elle soit, la fracture scolaire ne doit pas occulter l'apport culturel de l'école. Falardeau et Simard $(2015,21)$ insistent sur la complémentarité entre la culture première des apprenants et la culture scolaire, "seconde, réfléchie, incarnée dans des oeuvres, des systèmes symboliques, des pratiques, qui permet de dépasser ou de transcender le sens habituel de la vie quotidienne". Pour rendre effective cette complémentarité, les enseignants doivent à la fois rapprocher les pratiques scolaires des pratiques culturelles quotidiennes de leurs élèves et les aider à les différencier. S'agissant des deux types de discours qui nous intéressent, cette médiation doit s'effectuer simultanément sur les deux volets culturels travaillés par la discipline français ${ }^{1}$ : la langue et la littérature.

11 La grammaire "constitue [...] l'entrée dans une culture seconde de la langue » (ibid.) qu'elle transforme en objet de connaissance. Les types discursifs relèvent à la fois de la grammaire de la phrase (verbes, temps, modes, types de phrases et de propositions), du texte (schéma narratif, argumentatif) et du discours (stratégies argumentatives et narratives). Ce dernier niveau articule les structures de la langue et du discours aux opérations cognitives de la lecture. C'est donc la preuve que la connaissance grammaticale n'altère pas l'authenticité de la communication : «émanant d'un lecteur/ auditeur naîf, ou d'un linguiste-sémioticien bardé de concepts, l'interprétation conserve un caractère inévitablement hypothétique» (Adam 1985, 15). Adam en tire deux conséquences : le récit est aussi contraignant que l'argumentation : "De même que la force essentielle de la stratégie discursive publicitaire consiste à obliger le destinataire du message à entrer dans le jeu de l'argumentation en poursuivant les raisonnements, la force du récit consiste à obliger l'interprétant [...] à le compléter sans cesse » (Ibid.14). Et lorsqu'il a une fonction argumentative, il redouble l'impact de l'argument en lui donnant l'allure implacable du destin : «Le récit didactique tire son efficacité d'une leçon imposée à l'ouverture et à l'issue du déroulement du chapitre (ou de toute l'histoire) » (Ibid. 12).

12 L'école fait subir à la littérature le même sort que la grammaire : elle devient objet d'enseignement, ce qui l'éloigne de ses usages sociaux. Les stratégies de médiation culturelle déployées alors par les enseignants s'appuient à la fois sur les capacités du récit à modéliser les comportements humains et sur celles de l'argumentation à provoquer le questionnement intérieur :

Les thèmes que Rabelais va nous proposer ou que Ronsard amène dans tel poème, ce sont des choses qui ne vous intéressent pas, comme l'amour d'un gars pour une fille ? Ça ne nous intéresse plus? ». Alors là, ils sourient. Ils disent : «ben wa!» «Ah! ça vous intéresse, vous trouvez ça signifiant? Oui ? C'est important ? [....] 
Puis j'essaie de les amener à prendre conscience que ce qui se passait en 1500 quelque chose ; ça nous concerne encore aujourd'hui parce qu'on se pose les mêmes questions (Falardeau, Simard, op. cit. 23)

\section{L'emprise institutionnelle}

13 Le cadre institutionnel de l'école conditionne l'apprentissage par ses injonctions officielles et les normes de conduite tacites qui régulent les comportements des acteurs. Il est loin d'être uniforme. Reuter propose d'appeler configurations scolaires les formes prises par la forme scolaire dans les lieux où elle agit. (Reuter, 2012, 114).

Récit et argumentation sont concernés par l'emprise de la forme scolaire sur les apprentissages de façon générique en tant qu'objet didactiques imposés et dont l'apprentissage, soumis à évaluation, n'est pas sans conséquence pour l'image sociale de l'élève et son estime de soi ; et de façon spécifique, parce que, en tant que compétences communicatives, ils s'accommodent mal d'un apprentissage académique.

Enseigner les savoir faire discursifs ne peut se faire sans recourir à des situations authentiques, (ex: pour le récit, la confidence autobiographique; pour le discours, l'expression de l'opinion personnelle). L'injonction paradoxale entraîne alors une inégalité de traitement et de rendement entre les élèves qui savent ce qu'il faut dire et surtout ne pas dire, et ceux qui n'ont pas pris conscience du double fond de la pratique évaluative.

Les conséquences de cette aporie sont lourdes. En effet, nous avons montré (Biagioli, 2014), que, si certains élèves développent une écriture personnelle, le plus souvent narrative, via les réseaux sociaux et les sites de fanfictions, ils refusent toute parenté entre leur écriture privée et l'écriture scolaire :

$\mathrm{CH}$ : [..] tu n'aimes pas les cours de français ?//pourtant tu aimes la lecture et

l'écriture?

$\mathrm{S}:$ oui/mais la lecture et l'écriture : c'est pas du français

$\mathrm{CH}$ : pourquoi ?//parce que ça s'enseigne?

$\mathrm{S}$ : oui/pour moi la lecture et l'écriture c'est naturel/même si on l'enseigne/c'est

différent (Ibid. 162)

Le recours à une pédagogie non directive comme l'atelier d'écriture présente de nombreux avantages, notamment celui de convoquer l'argumentation authentique lors de la négociation du projet et des décisions d'écriture. Elle peut même s'articuler à l'écriture personnelle si l'atelier propose des consignes en lien avec les projets personnels des élèves. Mais une récente enquête (Biagioli, 2017), nous a alertée sur la réticence de certains élèves à montrer leurs productions scolaires aux autres. Raisons invoquées : «je sais que le mien est nul », « ils vont se moquer », « je n'aime pas être comparé », «parce que ça ne sert à rien, on n'est pas en compétition », " pas envie, je préfère le garder pour moi ». D'autres sont au contraire curieux de voir comment leurs camarades ont réalisé la consigne.

La forme scolaire amplifie la contradiction fonctionnelle des apprentissages discursifs en situation artificielle. Elle explique aussi pourquoi le français a préféré continuer la tradition de la médiation littéraire. Continuer un récit déjà écrit, disserter sur un propos d'auteur évite à l'élève d'exposer sa personne. Le souci de se préserver de la contrainte institutionnelle fait partie du métier d'élève comme de toute expérience professionnelle. 


\section{L'historique}

19 Présenter l'évolution des pratiques scolaires du récit et de l'argumentation suppose de distinguer d'abord enseignement primaire et enseignement secondaire, les demandes institutionnelles n'étant pas les mêmes. Les deux degrés n'ont été réunis qu'au moment de la démocratisation de l'enseignement secondaire, par la création du collège unique, en 1975. Tous deux ont un passé religieux. Les collèges jésuites - de 1540, date de la création de la Compagnie à 1773, date de sa dissolution par le pape Clément XIV - réalisent la première prise en charge institutionnelle de l'enseignement secondaire. Les écoles paroissiales qui se multiplient aux $\mathrm{XVII}^{\mathrm{e}}$ et $\mathrm{XVIII}^{\mathrm{e}}$ siècles peuvent être considérées (Giolitto, 2003) comme les ancêtres des écoles communales. Mais l'enseignement primaire est étatisé environ un siècle et demi avant l'enseignement secondaire, lorsque la loi Guizot oblige en 1833 toutes les communes à entretenir une école primaire de garçons et la loi Falloux en 1850 une école de filles. Et il s'émancipe plus rapidement de l'influence du latin, alors qu'initialement petites écoles et ordre jésuite sont tous deux des créations de la contre-réforme, dont un des enjeux est le maintien du latin comme langue de l'Église, alors que les protestants lisent la Bible en langue vernaculaire.

\section{Avant le collège unique}

La différence de tempo dans l'évolution de la langue d'enseignement au primaire et au secondaire va de pair avec la différence des cultures pédagogiques. En effet, si le latin est concerné dans les deux cas, celui de l'école paroissiale est le latin liturgique. Il est mémorisé avec sa traduction pour faciliter l'accès au sens des textes, ce qui entraîne des modalités de communication paraphrastiques et interrogatives (sur le modèle du catéchisme). Tandis que le latin du collège jésuite est le latin profane des humanités classiques (plus païennes que chrétiennes), attachées aux valeurs humanistes de la Renaissance et à un système d'enseignement basé sur la rhétorique, c'est-à-dire sur les apprentissages discursifs. Il est écrit et parlé. Ceci entraîne des usages très différents du récit et de l'argumentation, qu'ils soient didactiques, lorsqu'on les enseigne, ou pédagogiques, lorsqu'on les emploie pour enseigner.

21 Pour présenter le récit et de l'argumentation dans l'enseignement primaire, nous avons choisi un manuel de la fin du XIX siècle: le Cours Elémentaire de lecture expliquée et de composition française (Causeret, 1891). Fils d'instituteur, Causeret fait des études de lettres au lycée, puis à l'université et obtient l'agrégation de grammaire. Répétiteur en collège puis en lycée, il devient professeur de rhétorique puis, passant de l'enseignement à la haute administration, devient inspecteur d'académie en 1885 et recteur en 1907. En 1886, il soutient une thèse : Etude sur la langue de la rhétorique et de la critique littéraire chez Cicéron , et publie en 1889 une étude : 3 fabulistes: Esope, Phèdre et La Fontaine (Condette, 2006, 102-103).

22 Ces précisions éclairent la transposition didactique de la culture humaniste et de la formation rhétorique à laquelle il se livre dans son manuel. Le titre fait référence à deux genres scolaires emblématiques du secondaire : l'explication de texte et la composition française. Le livre s'adresse aussi bien aux élèves de la division élémentaire des écoles primaires qu'à ceux des classes primaires des lycées et collèges. On y trouve donc les prémices d'un changement qui, quatre-vingt ans plus tard, fera du primaire, à l'origine 
école du peuple, le premier niveau de l'enseignement pour tous. Le choix des textes repose sur un souci d'édification, dans lequel la morale mondaine prend le relais de la morale religieuse. Pour le récit, on trouve un choix de fables, les plus connues de La Fontaine: La cigale et la fourmi, Le renard et le bouc, mais aussi des fables de Fénelon, Florian, Arnaud, Le Bailly, Lachambaudie, et d'anecdotes édifiantes (Mme de Genlis, Voltaire). Les textes argumentatifs sont pour la plupart des poésies didactiques qui développent des arguments à l'appui d'une prescription morale.

Le questionnaire de guidage de la lecture est surtout destiné à faire paraphraser le texte aux élèves, car, comme en avertit l'auteur dans la préface :

Expliquer, c'est [..] déployer, étendre et développer ce qui était plié, afin de le faire bien voir dans toutes ses parties. Les questions posées [...] ont précisément pour objet de décomposer chacun de ces morceaux [choisis] en autant de parties qu'il y a d'idées essentielles, et de permettre ainsi [...] de saisir la pensée dominante (ibid., 8)

L'exercice de composition qui suit le questionnaire consiste parfois simplement à répondre par écrit à chaque question et disposer en paragraphes les réponses obtenues. Ce peut être aussi une transposition dans un univers familier des élèves, soit celui de leur milieu (métiers, famille), soit celui de l'école. Ainsi, suite à une anecdote mettant en scène Louis XIV refusant d'admettre qu'il a triché au trictrac, on s'appuie sur les Fables de La Fontaine pour demander d'imaginer une contestation entre le lion et l'ours au cours d'une chasse, arbitrée par le renard. Pour l'argumentation, on propose de faire l'éloge du père, après avoir lu un poème faisant celui de la mère. Mais on fournit les arguments : «Dites pourquoi vous aimez votre papa: I. 媐 Votre papa travaille rudement pour vous élever, II. 圈 Il vous comble de caresses et de jouets. III. 㡠 Sa physionomie quand vous êtes

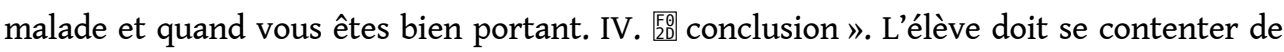
les endosser, en modifiant l'énonciation. De ce fait, il est contraint d'admettre les arguments qui sont présupposés sans pouvoir les discuter, et n'a pas davantage conscience qu'il fait un exercice de grammaire en passant de la $2^{\text {ème }}$ à la $1^{\text {ère }}$ personne.

Nous trouvons dans l'ouvrage d'Houdart-Mérot sur La culture littéraire au lycée depuis 1880 (1998), de quoi compléter au niveau du secondaire ce qui vient d'être établi pour le primaire. Principales continuités repérées. D'abord la langue: 1880 est une date symbolique qui marque le remplacement de la composition latine par la composition française. Le français cesse d'être seulement la langue de traduction du latin et du grec. Ensuite une pratique rhétorique, basée sur l'elocutio, recourant à l'amplification, la réduction et la substitution. Principales différences: la longueur et la complexité des textes demandés et l'omniprésence de la culture littéraire. En outre, le choix des textes à partir de 1925 s'affranchit des considérations morales (ibid. 85), et, à partir des années 60 (ibid.162-163) s'ouvre à la littérature d'idées ainsi qu'au roman, chargé de donner aux élèves le goût de la lecture.

Houdart-Merot confronte aux Instructions officielles un choix de devoirs corrigés qui font mesurer le décalage entre curriculum prescrit et curriculum appris et renseignent sur les pratiques de lecture littéraire des élèves et les pratiques évaluatives des enseignants. Certains, parmi lesquels l'amplification par Proust (1887) d'une anecdote historique sur Christophe Colomb (ibid., 249) sont des récits. La plupart relèvent de l'argumentation, indirectement (ex: «Lettre d'un ami à Cicéron pour le consoler de la mort de sa fille » (1915), ou directement comme les dissertations littéraires et générales, ou les commentaires composés. 


\section{Après le collège unique}

27 Les auteurs de manuels du collège unique veulent démocratiser la culture littéraire du secondaire supérieur tout en respectant l'objectif du secondaire inférieur : assurer une formation de base à tous ceux qui quittent l'école en fin de $3^{\text {èmez }}$. Mots et Merveilles $5^{e}$ (Magnard, 1982) opte pour une double approche par genres et par thèmes, à la fois plus littéraire et plus réflexive mais aussi plus personnelle qu'au primaire. Des textes sans questionnaire sont réservés " pour une lecture buissonnière » (ibid.2-3). Pour familiariser avec la littérature : des témoignages d'écrivains sur leur métier, plutôt descriptifs, mais contenant des anecdotes, ou des récits itératifs. Pour rester ancré dans la vie : des textes informatifs renseignant sur certains sports, le travail en usine, la vie de tribus amazoniennes, etc. Les textes narratifs sont des textes littéraires patrimoniaux (L'̂́le des esclaves de Marivaux, Fables de La Fontaine, Les Châtiments d'Hugo), des récits symboliques (contes, mythes), ou des textes autobiographiques.

28 Le chapitre $D u$ récit au roman, consacré aux régimes de vérité du récit, juxtapose des extraits de fictions et de récits authentiques autour d'un thème (ex : La planète des singes de Boulle et un compte-rendu d'éthologue: L'histoire de Nim, le chimpanzé qui parle, 222-235). L'expression écrite est elle aussi différenciée : «Imaginez un nouvel épisode de La planète des singes. Transposez-le en jeu dramatique. 2. Vous écrivez à un(e) camarade et vous lui faites part de L'histoire de Nim et de vos réactions à la lecture de ces documents ». L'organisation narrative est étudiée à partir de la BD: "Comment les vignettes s'ordonnent-elles entre elles ? Retrouvez le déroulement de l'histoire », 119).

Pas de textes argumentatifs. En revanche, une stratégie d'éveil à l'argumentation à partir de textes informatifs, pour déconstruire les préjugés («causerie-débat: En quoi ces documents modifient-ils l'image conventionnelle de l'Indien à travers la bande dessinée et le western?», 115) ou prendre conscience d'une problématique sociale («Quels sentiments, quelles réflexions naissent en vous en analysant ces textes sur les enfants prolétaires ? Étiez-vous au courant, par des lectures, des conversations, des films, de ces problèmes de l'enfance dans les pays non occidentaux? ", 187).

Si la volonté de hisser le primaire au niveau du collège est commune à tous les manuels de collège, celle d'ouvrir le lycée à l'esprit du collège ne se retrouve que dans une seule collection de manuels de lycée, Littérature et langages (1973-75) dirigée par H. Mitterand.

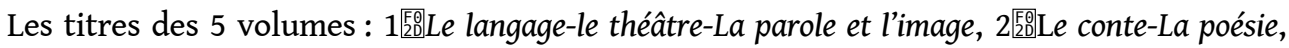
3 㜯Le roman-le récit non romanesque-Le cinéma, 4 㡠La littérature et les idées, 5 㡠 Thèmes et langages de la culture moderne, montrent une véritable continuité avec les objectifs de Mots et Merveilles. Quinze ans après, Mitterand reviendra au modèle traditionnel des siècles littéraires avec la collection Littérature Moyen-Âge-XVIe, XVII $, X^{e} I_{I I}^{e}, X_{X}{ }^{e}, X X^{e}$, textes et documents. Ces deux collections emblématisent l'hésitation entre deux modèles d'enseignement de la littérature : celui narratif de l'histoire littéraire et celui descriptifexplicatif de la sémiotique littéraire. Le premier, fruit d'une tradition qui remonte à $G$. Lanson, n'a jamais été déconstruit sauf, épisodiquement, au niveau de l'enseignement supérieur (cf. Biagioli, 1995, 2013). Sa romancisation facilite la mémorisation des repères historiques. Le second, plus difficilement transposable en raison du nombre de sciences humaines qu'il convoque, joue un rôle de contrepoids, et assure le lien entre la littérature scolarisée et la littérature pratique sociale de référence, ainsi que les autres discours sociaux. 


\section{La progression didactique}

31 En tant qu'objets didactiques, narration ${ }^{3}$ et argumentation sont des compétences discursives, descriptibles sous l'angle de la compréhension /production, et de l'intermédialité (écrit/ oral / image/ son, etc.). La progression curriculaire est un produit de la transposition didactique qui analyse l'objet à enseigner en une série d'éléments ordonnés du plus simple au plus complexe. Depuis une quinzaine d'années ce modèle linéaire a cédé la place à un modèle spiralaire qui propose d'emblée une version simplifiée mais déjà complexe de l'objet. Narration et argumentation s'enseignent par quatre voies, toutes quatre complémentaires : la pratique empirique (essais et erreurs), la théorie (narratologie, grammaire de texte), l'application de la théorie pour modéliser la pratique (lecture analytique ou algorithme d'écriture), et le retour sur la pratique qui produit l'expérience (autoconfrontation, instruction au sosie).

\section{De la maternelle à l'école élémentaire}

À la maternelle, on parle de savoirs pré-disciplinaires, plutôt que disciplinaires (Daunay, Delcambre, 2007, 38-39). Pour autant, récit et argumentation sont pratiqués quotidiennement.

Les enfants apprennent d'abord à maitriser le langage en situation, en décrivant ce qu'ils font, puis le langage d'évocation qui met en jeu la mémoire pour le récit au passé, et la prévision pour le récit au futur. Nous donnons un exemple de ce dernier, emprunté à une ingénierie couramment employée avec les moyennes sections (3-4 ans): le séjour de week-end de la mascotte de classe. Les enfants amènent à tour de rôle la peluche chez eux, et doivent dire avant ce qu'ils vont faire avec elle, et après ce qu'ils ont fait. La première phase, qui nous intéresse ici, est une construction fictionnelle, alors que la seconde est un récit authentique. Étayée par les questions de l'enseignante, l'élève esquisse un programme :

Qu'est-ce que tu aimerais faire le week-end prochain? - Ben je vais aller au parc

avec lui - Qu'est-ce que tu vas faire au parc? - j'vais le faire jouer au foot. - Oui/

ensuite? - Ensuite, je vais le faire glisser dans le toboggan.

puis digresse vers des thèmes, secondaires pour le récit mais primordiaux pour elle : « Tu crois qu'elle va vouloir jouer avec lui Roxane [sœur de l'élève]? - Oui/ en plus j'ai la trottinette et le vélo Titi/j'ai un grand vélo à deux roues ».

Deux caractéristiques émergent, documentées par les narratologues. La première est la nature coopérative de la narration, moins sensible à l'écrit mais patente à l'oral. « La mise en intrigue dépend d'une communication [...] qui retarde stratégiquement le dévoilement d'une information essentielle afin d'intriguer un destinataire qui accepte généralement de se prêter au jeu de l'intriguant », (Baroni, 2010). On la retrouve dans : « Il va prendre le ti déjeuner avec moi - Il va prendre le petit déjeuner avec toi ! - dire à maman qu'elle lui donne un ti déjeuner//i va boire du chocolat chaud/i va manger du sucre dans le chocolat ».

La seconde est la sensibilité aux bifurcations narratives. «Bien avant de s'intéresser aux médias numériques ou aux fictions interactives, certains narratologues avaient déjà intégré dans leurs modèles une forme minimale d'interactivité en évoquant les virtualités 
de l'intrigue, que l'interprète scénarise au cours de sa progression dans le texte ", (Marti, Baroni, 2014). Ici la virtualisation intervient lorsque l'élève imagine les réactions des membres de sa famille : «j'vais faire peur à ma maman et à mon papa. - Et à ta sœur? - Oui : Roxane elle va rigoler//ou peut-être elle va pas rigoler ».

L'argumentation en maternelle sert à faciliter le langage d'évocation, à se familiariser avec les règles de l'intervention publique et à se décentrer. Sa pratique-phare est le débat philosophique, embrayé par une question à portée définitionnelle (ex : qu'est-ce qu'être heureux? être un bébé? sommes-nous tous pareils?), une image favorisant les hypothèses interprétatives, ou l'exploitation d'un désaccord intervenu en classe. L'enseignant y joue le même rôle que dans l'étayage du récit : il questionne, relance, et gère le débat.

Au cycle 2 de l'école élémentaire l'apprentissage de la narration se fait à l'oral. Le CP met l'accent sur la compréhension, le CE1 sur la production. En fin de cycle l'élève doit produire un récit «structuré [...] et compréhensible pour un tiers ignorant des faits rapportés ou de l'histoire racontée »4. En lecture, il doit «identifier les personnages, les événements et les circonstances temporelles et spatiales ». L'argumentation est uniquement travaillée à l'oral, avec le débat, « écouter et donner un point de vue en respectant les règles de la communication », et la « lecture dialoguée » qui présuppose la perception de la logique argumentative du texte lu.

La notion d'argument n'apparaît qu'au cycle $3^{5}$ : «échanger, débattre : [...] rester dans le sujet, situer son propos [...], apporter des arguments, mobiliser des connaissances) ». Le débat interprétatif qui invite à "expliciter des choix de lecture, des préférences " instaure entre argumentation et lecture littéraire une relation durable. La narration devient un simple outil. À l'oral elle est prérequise par le débat : il faut pouvoir « raconter de mémoire une œuvre lue ", avant d'en discuter. À l'écrit, où l'élève doit «maîtriser la cohérence des temps dans un récit d'une dizaine de lignes", elle sert à contrôler l'acquisition des micro et médio-structures grammaticales. Se met alors en place un principe de progression qui se poursuit aux niveaux suivants. L'oral précède l'écrit, la compréhension la production, la narration l'argumentation. Toutefois, en décloisonnant les activités, certaines ingénieries parviennent à dépasser les attentes de fin de cycle. Par exemple, le projet d'écriture théâtrale fait d'abord travailler le récit avec le scénario, puis l'argumentation avec les dialogues, et les deux avec la représentation car l'acteur fait le chemin inverse de celui de l'auteur: il se sert des dialogues pour s'immerger dans l'univers fictionnel. Le dispositif est facilité lorsque l'enseignant part d'un hypertexte comme Don Quichotte et qu'il initie lui-même la réécriture (Peyrou, 1999).

\section{Du collège au lycée}

La dénomination de l'explication de texte réfère explicitement à l'argumentation. La mise en place de la lecture littéraire réflexive au collège marque un changement de statut de la narration et de l'argumentation. Ce ne sont plus seulement des objets ou sous-objets didactiques. Elles deviennent des outils de compréhension, d'interprétation et d'évaluation des connaissances. Dans ces rôles, elles s'appellent et se complètent, formant un cercle vertueux, mais qui reste implicite, puisque ni les enseignants ni les élèves ne conscientisent ce double statut.

41 Deux méthodologies de l'explication de texte se distinguent. L'une, en gros celle des manuels, dissocie compréhension et interprétation. Quel que soit le support, texte ou 
image, l'algorithme de lecture fait se succéder lecture informative et lecture interprétative. Ex : «Observer les images ${ }^{6}$. 匰 Identifiez la nature des supports et leur

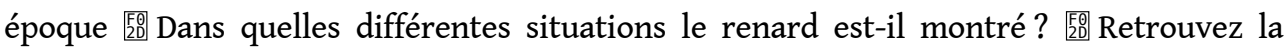
visée de chaque image : illustrer, orner, faire rire?». (Rives Bleues $5^{\mathrm{e}}$, Hatier 2010, 171). Parfois on demande à l'élève d'exprimer sa réaction, mais en la justifiant : "Aimez-vous cette image ? Justifiez votre réponse " (ibid. 95), ou d'imaginer les réactions possibles : «Observez l'affiche [...] et lisez le texte. Quels éléments peuvent donner l'idée d'aller voir le film?» (ibid. 175). Le lecteur scolaire est contraint de se justifier comme si l'école redoutait l'épanchement émotionnel, préférant lui substituer son explication par le fonctionnement des codes sociaux que sont les genres et les registres littéraires.

L'autre méthodologie, en gros celle des enseignants innovants, adopte le parcours inverse, part du ressenti de lecture avec un embrayeur comme: «qu'est-ce qui est important à votre avis dans ce livre?» (Weisser, 2006, 37) et grâce à l'échange entre pairs, fait émerger la diversité des réactions. Elle traite l'explication de textes comme une activité de résolution de problèmes, utilise la narration de la démarche pour la faire conscientiser, et l'analyse comparée des solutions pour faire saisir leur cadre de validité.

43 Au lycée, la technicisation de la lecture littéraire provoque la crise de la lecture analytique dans les années 1980-2000 (Boissinot, 2001, 187). Retoquée en lecture méthodique, l'explication de texte se fonde désormais sur des catégories plus larges que les figures de style: genres, registres, stéréotypes, mais son principal mérite est de familiariser les élèves avec la " coopération interprétative » chère à Eco, en resituant le texte dans un horizon d'attente et en montrant la façon dont l'auteur se sert de ce dernier pour jouer avec le lecteur. Toutefois cette démarche ne permet pas de répondre aux attentes d'un public scolaire en manque d'immersion narrative primaire et étouffé par l'injonction justificative, car, ainsi que le rappelle Langlade $(2001,149)$ «l'étude méthodique des textes perdrait tout son sens si elle ne s'articulait pas avec une expérience intime, émotionnelle et affective des œuvres », une expérience qui ne peut s'exprimer qu'à travers le récit, et qui a donné lieu à une nouvelle génération d'outils didactiques : journal de lecture, carnet de bord.

Narration et argumentation se retrouvent non seulement dans les techniques mises au service de la lecture mais aussi dans les textes étudiés et les textes produits, associés dans une didactique de la production écrite qui privilégie l'écriture hypertextuelle. Le rapport entre genre de textes et type de discours a été abondamment traité par les poéticiens et les didacticiens. Rappelons que l'articulation des textes aux discours a fait basculer la typologie textuelle de l'histoire littéraire vers la grammaire du texte et du discours, et permis l'articulation dans un schéma didactique cohérent : la séquence décloisonnée, de la lecture, de la production écrite et de la maîtrise de la langue. Le genre littéraire scolaire est un pur produit de ce bricolage, un «savoir de compromis », (Denizot, 2005, 43).

Historiquement, l'école a réhabilité le genre au moment même où il était complètement récusé par la pratique sociale de référence. Une remise en cause commencée avec Hugo qui s'est poursuivie tout au long du $\mathrm{XIX}^{\mathrm{e}}$ et $\mathrm{du} \mathrm{XX}^{\mathrm{e}}$ siècle. Pourtant l'analyse des mouvements de contestation littéraire, notamment du Nouveau Roman, montre qu'à l'instar de l'auteur, le genre ne meurt pas mais sert de repère pour inventer, métisser, déconstruire, renouveler.

Le genre s'articule aux types de discours narratif, descriptif, argumentatif, explicatif, grâce à deux notions : la dominante, élaborée par les formalistes russes (Combe, 1992, 
117) qui permet de décrire l'inclusion des structures textuelles d'un genre par un autre, et la tonalité (ou registre) qui rend compte des transferts d'effets entre genres (ibid. 21). Ce modèle qui équilibre effets de forme et effets de sens convient au lycée, où il guide aussi bien la formulation des hypothèses de lecture que leur exemplification. Il y coexiste avec un autre modèle, beaucoup plus ancien, dont Combe (ibid., 145) constate la permanence : la trilogie épique, lyrique et dramatique, qui limite les genres au seul récit et dont la transposition didactique dans les trois archi-genres: roman, poésie, théatre, rythme l'alternance des séquences. Cette cohabitation se remarque à de (rares) contradictions internes du discours didactique (ex : l'inclusion d'un extrait de La Chanson de Roland dans une section intitulée Le personnage du roman à l'épreuve, (La vie en toutes lettres 1 ère, Hatier, 2016, 78). La déformation prototypique du récit qui finit par représenter toute la littérature bloque à la fois l'identification des genres narratifs non littéraires et celles des autres genres, notamment argumentatifs.

La didactisation de l'écriture ressemble en tous points à celle de la lecture, dont elle n'est souvent que le prolongement. La progression part du récit en $6^{\mathrm{e}}$, enrichi en $5^{\mathrm{e}}$ avec la description, en $4^{\mathrm{e}}$ avec le dialogue, en $3^{\mathrm{e}}$ avec le point de vue, et atteint l'argumentation en $3^{\mathrm{e}}$, sous deux formes: " présentation d'une prise de position étayée par un argument concret [...] et un argument abstrait [...]; présentation de plusieurs opinions sur une question" (Programmes et accompagnements, juillet 1999, 1577). Là aussi deux méthodologies s'affrontent. Les I.O. propose des exercices fréquents et ciblés (sur des structures grammaticales), tandis que certains auteurs de manuels (cf. la collection Lire écrire ensemble d'Oriol-Boyer, Sauzeau, Hatier, 1993-96) associent pédagogie par objectifs et pédagogie de projet pour offrir aux élèves une authentique expérience d'écriture.

On a pu penser que le camp de la créativité avait gagné une bataille lorsque l'écrit d'invention a fait son apparition dans les épreuves du baccalauréat, mettant fin à la coupure entre un collège où l'on écrivait des textes, et un lycée où l'on n'écrivait que du métatexte critique. Or le terme d'invention marque surtout un retour à la rhétorique restreinte (Biagioli, 2016, 609-611). Des genres scolaires désuets comme la lettre fictive entre deux auteurs, l'article de presse fictif sur la première d'une pièce, sont réapparus, à côté de formes plus authentiques comme la continuation, ou bien ouvertement artificielles comme la réécriture antonymique oulipienne.

La progression didactique multiplie les contradictions et les hésitations à l'égard des deux compétences et se montre perméable aux stéréotypes qui les opposent: narration plus facile et plus spontanée, argumentation plus difficile et plus technique. Dans le déroulé du cursus, l'argumentation monte en puissance, mais une puissance contenue par l'application à la littérature et la fonction évaluative. Les genres argumentatifs purs sont presque ignorés, d'autant que l'échec de la didactique de l'oral a limité la possibilité de former à l'argumentation orale. En les restreignant à leurs fonctions scolaires, le dispositif d'apprentissage prive les deux types discursifs de visibilité.

\section{Récit et argumentation dans la refondation de l'école}

Cette dernière partie n'a pas pour but d'ajouter un nouvel épisode historique à notre étude mais de la compléter en prenant en compte un paramètre capital quoique rarement étudié : l'impact des politiques éducatives sur l'enseignement. 
51 La réforme de 2013 a touché les deux types discursifs aux niveaux :

- de l'enseignement, dont elle change radicalement le système d'évaluation;

- du projet éducatif, centré sur le vivre ensemble, le débat citoyen et donc l'argumentation;

- du projet culturel, articulé à la fois aux ressources des collectivités territoriales en matière d'éducation artistique, et à une refondation du récit national.

\section{Le passage aux compétences}

52 Narration et argumentation sont des compétences transversales dont chaque discipline assure un apprentissage ciblé sur les situations qu'elle travaille. C'est pourquoi les didacticiens ont décrit les disciplines scolaires comme des " communautés discursives » (Bernié, 2002) et fait de l'analyse des interactions verbales en classe une des méthodologies de la didactique comparée. Le passage du modèle d'évaluation par objectifs au modèle d'évaluation par compétences en 2013 ne change rien en apparence à ces apprentissages discursifs sauf ... qu'il les rend implicite. Il met l'accent sur des compétences pluridisciplinaires scolaires, préfiguration des compétences professionnelles générales attendues par les employeurs : «lire et comprendre l'écrit », "s'exprimer à l'oral en continu et en interaction", «maîtriser l'expression de sa sensibilité et de ses opinions, respecter celles des autres $»^{8}$.

Que reste-t-il de la progression curriculaire du français au collège ? Curieusement, on la retrouve intacte....dans la progression en langue étrangère, en compréhension (récit:

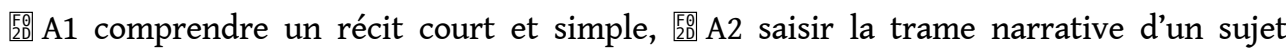
portant sur un sujet familier ou déjà connu; argumentation: 區B1 comprendre les grandes lignes d'un débat contradictoire, suivre les points principaux d'une discussion d'une certaine longueur sur un sujet familier), et en production (récit: 媐 A2 écrire un court récit, 医 B1 écrire un récit construit; argumentation: 㡠 B1 défendre un point de vue sur un sujet d'actualité ou déjà connu).

C'est que l'on ne parle plus d'une discipline mais de "toute discipline intégrant un enseignement en langue étrangère (ou régionale) » (op. cit., 4) ${ }^{9}$. Pour le reste, narration et argumentation sont délocalisées dans tous leurs usages : évaluatifs, expressifs, culturels.

Le français est écartelé entre ses «contributions essentielles aux 5 domaines du socle commun » (Langages pour penser et communiquer, Méthodes et outils pour apprendre, Formation de la personne et du citoyen, Systèmes naturels et systèmes techniques, Représentations du monde et de l'activité humaine), et les 4 thèmes de sa nouvelle progression au cycle 4 (Se chercher/se construire; Vivre en société, Participer à la société; Regarder le monde/inventer les mondes; Agir sur le monde) ${ }^{10}$. Il y retrouve certaines de ses anciennes marques: le récit autobiographique (Se raconter/se représenter) en $3^{\mathrm{e}}$, littératures d'idées (Dénoncer les travers de la société) en $4^{\mathrm{e}}$, mais la thématique psycho-sociale retenue paye son harmonie avec les finalités de la refondation d'un manque de différenciation entre savoirs, savoir-faire et savoir-être.

\section{Le débat citoyen, entre rhétorique rationnelle et rhétorique émotionnelle}

Des 5 domaines du socle commun au cycle 4 , le plus fédérateur a priori est le $3^{\text {ème }}$ : Formation de la personne et du citoyen. En soi il n'a rien d'original, puisque tous les 
systèmes scolaires de par le monde affichent le même. Mais il faut compter avec le contexte scolaire, socio-économique et géopolitique. La France a obtenu de mauvais résultats de la France aux enquêtes PISA 2009 et 2012. Elle entre en récession économique officiellement en 2009. Les attentats terroristes de mars 2012 montrent l'impuissance de la société française et de son école à socialiser un enfant marqué par une situation familiale chaotique, un décrochage scolaire précoce et un environnement communautarisé (Mohamed Merah est franco-algérien et a été scolarisé en France).

Le ton de la présentation est péremptoire : «La formation de la personne et du citoyen relève de tous les enseignements et de l'enseignement moral et civique ». On veut éviter que la création de la nouvelle discipline enseignement moral et civique n'incite les autres à se défausser sur elle de l'effort de formation. On cible les contributions plutôt par familles de disciplines que par discipline, évoquant :

Les connaissances scientifiques et techniques qui permettent d'accéder à la vérité et à la preuve, de la différencier d'une simple opinion, de comprendre les enjeux éthiques des applications scientifiques et techniques; le respect des règles et la possibilité de les modifier ; les savoirs littéraires et historiques indispensables à la compréhension du sens de la citoyenneté, de la place de l'individu dans la société et du devoir de défense, (ibid., 222).

Un raccord intervient à la fin de la présentation de chaque programme disciplinaire. Pour le français c'est :

Enfin, l'enseignement du français contribue fortement à la formation civique et morale des élèves, tant par le développement de compétences à argumenter que par la découverte et l'examen critique des grandes questions anthropologiques, morales et philosophiques soulevées par les œuvres littéraires (ibid., 227).

Nous ne nous appesantirons pas sur les représentativités respectives de la narration et de l'argumentation dans ce programme. Il n'est guère surprenant que dans des situations de violence civile, l'argumentation et la réflexion sur l'endoctrinement de ceux qui mettent en danger la cohésion sociale soient jugées plus sérieuses que la narration, puisqu'elles font appel à la raison. C'est ce qui s'est déjà passé lors de la refondation de la rhétorique ancienne après la $2^{\text {nde }}$ guerre mondiale. Toutefois, pour déconstruire les mécanismes de l'endoctrinement, il faudrait, tout en réintroduisant une initiation à la logique formelle, base indispensable à l'évaluation de la recevabilité des arguments dans la rhétorique rationnelle ${ }^{11}$, s'inspirer des récentes avancées de la recherche sur la rhétorique émotionnelle (Plantin, 2011), ce qui permettrait de renouer les fils avec la narration, puisque la rhétorique émotionnelle repose sur l'empathie, qui elle-même repose sur l'immersion fictionnelle.

60 Ce qui nous ramène à la fonction médiatrice de la littérature à l'école, qui permet de parler des problèmes éthiques et sociaux dans un univers alternatif, dérivé de l'univers de référence, mais qui peut être modifié et questionné sans que cela nuise à sa stabilité.

\section{Le retour du récit national}

61 Même s'il n'est encore complètement passé à l'évaluation par compétences, le lycée été impacté par la refondation de 2013. Les 4 objets d'étude de 2010 ont été conservés, 3 consacrés aux archi-genres narratifs roman, théâtre, poésie, un seul au « genres et formes de l'argumentation ». Maintenu également le dédoublement de cette thématique entre l'approche de la $2^{\text {nde }}$ centrée sur l'histoire littéraire ( Le roman et la nouvelle au XIX ${ }^{\mathrm{e}}$ siècle : réalisme et naturalisme, La tragédie et la comédie au XVII ${ }^{\mathrm{e}}$ siècle : le classicisme, 
Genres et formes de l'argumentation: XVII et XVIII ${ }^{e}$ siècle »), et celle de la $1^{\text {ère }}$ centrée sur l'anthropologie littéraire (« Le personnage de roman, du XVIIe siècle à nos jours, Le texte théâtral et sa représentation, du XVIIe siècle à nos jours, Écriture poétique et quête de sens, du Moyen Âge à nos jours »), lointain souvenir de la progression curriculaire de l'enseignement jésuite, dans laquelle la classe de rhétorique était l'antichambre de la classe de philosophie.

La différence est plutôt dans la présentation des objets. Si l'on compare les tables des matières de deux manuels de $1^{\text {ère }}$ du même éditeur Hatier, réalisés par la même équipe avant : Terres littéraires (2011), et après : La vie en toutes lettres (2016), la réforme, on s'aperçoit que l'ordre des questions a changé. On commence par «Le personnage de roman », et on termine par "L'argumentation: la question de l'homme», alors que le précédent manuel commençait par «La poésie : la quête du sens »et terminait par «Le texte théâtral ». Récit et argumentation polarisent les activités autour d'une idée-force : la fonction sociale d'une littérature qui permet projection et identification mais aussi prise de conscience. Ceci se trouve confirmé par un changement dans la présentation du personnage de roman, vu à travers le regard que la société porte sur lui dans une section "Quand la société juge le personnage» (La vie en toutes lettres, 2016, 4-11), et l'introduction dans cette séquence d'une œuvre intégrale permettant de découvrir «Le goût du risque chez les jeunes ». Le manuel précédent proposait des groupements de textes centrés sur l'histoire du genre: 3 Héros et anti-héros, La femme dans le roman, Nouveaux narrateurs, nouveaux personnages », Terres littéraires, 2011 7-9).

La seconde différence réside dans l'articulation forte des trois parties du manuel: littérature, outils, méthodes, au moyen de questions sous-titrant les chapitres de littérature et préparant l'élève aux pistes de réemploi dans les sujets de dissertation ou de commentaire composé.

La comparaison des titres des deux manuels prouve qu'en 5 ans on est passé de l'exploration de la littérature comme une terre lointaine, à une apparente intégration de la littérature à la vie à partir des conduites humaines qu'elle met en scène, mais en réalité à un prédécoupage argumentatif des textes littéraires préparant leur réemploi dans les exercices du baccalauréat. On est donc passé du "roman de l'histoire littéraire » (Biagioli, 1993) un roman dont les structures explicitement stéréotypées avaient été conçues pour servir de repères à l'apprentissage historique, à un autre, beaucoup moins innocent, qui est le roman de la littérature scolaire, une littérature présentée comme la panacée universelle aux maux des sociétés modernes. Conditionné a priori par l'appareil argumentatif des exercices du baccalauréat, qui n'est pas une mise en débat, mais la reformulation d'arguments déjà fournis sur des thèmes déjà connus, ce roman-là se dérobe à toute interrogation, et se coupe des racines vives de l'argumentation alors même qu'il prétend y former. Comment s'est-il édifié ?

Le document Ressources pour le lycée général et technologique de 2013 étend les genres argumentatifs à toute la littérature "sans pour autant considérer que tout texte est argumentatif », au prétexte que «les Belles-Lettres ont longtemps tenu le rôle de boussole axiologique, en posant au fil des siècles la question des valeurs de l'homme dans et face au monde. $\aleph^{12}$. La refondation des Belles-Lettres réunissant l'histoire, la philosophie et le français en une croisade commune pour la propagation des valeurs humanistes éternelles est une projection sur le futur d'un passé lointain et fantasmé de la discipline. Elle occulte, outre la spécificité de la littérature comme production culturelle, les erreurs politiques et éthiques de certains écrivains et l'utilisation de la littérature elle- 
même à des fins de propagande. Comme Bruner (2002, 91), nous sommes amenés à constater que : « les histoires uniques, stéréotypées, n'en continuent pas moins d'imposer un durcissement ontologique aux diverses versions du monde réel ». C'est sur ce point qu'il convient de se montrer vigilant, même et surtout si ce roman de la littérature scolaire vient étayer le roman national. Un travail de déconstruction s'impose pour n'en faire qu'un des récits possibles de la littérature et de son enseignement.

\section{Conclusion}

Le couplage du récit et de l'argumentation est en soi un parti-pris argumentatif. Il laisse de côté les autres types de discours : descriptif, explicatif, injonctif, ce qui, d'un point de vue didactique, n'est pas satisfaisant, car, devenus sous-ensembles des deux types cardinaux, ils ne peuvent être appréciés dans leurs fonctionnements propres.

Toutefois, nous avons pu constater que l'ancienneté de ce couplage en fait une pierre de touche de l'histoire et de l'épistémologie du français. Il y a un plus d'un quart de siècle, J.F. Halté $(1992,45)$, remarquait que cette discipline se prêtait peu à une conception topdown de la transposition didactique, parce qu'elle était issue de la rhétorique et que cette dernière était issue de l'expérience professionnelle, donc plutôt bottom-up. Non seulement la rhétorique comme art d'agir par la parole a produit les techniques d'enseignement des discours que l'école a institutionnalisés, mais elle lui a de surcroît légué ses contradictions didactiques: comment maintenir l'équilibre entre forme et contenu, improvisation et manipulation, rationnel et émotionnel, etc.

L'enseignement d'une compétence professionnelle suppose une bonne connaissance des savoirs d'action, leur modélisation en schèmes opérationnels, leur exercice dans des situations de simulation, et une alternance entre l'enseignement théorique et la découverte $\mathrm{du}$ milieu professionnel. C'est, nous l'avons vu, ce que les nouveaux programmes, avec l'institutionnalisation des compétences pluridisciplinaires et des projets ouverts sur la société, essaient de mettre en place. Ceci devrait avoir pour effet de remettre le récit au centre du dispositif de formation à parité avec, et comme source possible de l'argumentation ; un récit qui permet à l'apprenant de s'approprier ce qu'il vit, et de lui donner sens, qu'il le lise ou le produise, pour imaginer ou pour comprendre comment il apprend. Pour la rhétorique antique, le récit n'est qu'une preuve parmi d'autres, une preuve émotionnée qui agit sur les sentiments, par le biais de l'identification. Ce que voudrait apporter l'approche scolaire de la littérature, c'est une plasticité de conduite qui permettrait de goûter les plaisirs du texte, tout en aiguisant son jugement. Il semble que cela ne soit possible que si l'on accepte de reconnaître la nature profondément ambivalente du récit, ce qui implique, comme l'a montré Paul Veyne (1983, 28) à propos des mythes, que l'on tienne compte de la pluralité des régimes de vérité sans les confondre. 


\section{BIBLIOGRAPHY}

Adam J.-M. (1985). Le texte narratif. Paris : Nathan.

Baroni R. (2010). Le temps de l'intrigue. Cahiers de Narratologie. (18).

Bernié J.-P. (2002). L'approche des pratiques langagières scolaires à travers la notion de " communauté discursive »: un apport à la didactique comparée? Revue française de pédagogie, (141), Vers une didactique comparée. Paris : INRP, 77-88.

Biagioli N. (2017). Interdidactique et didactique du français : quels (r)apports ? quels enjeux. Recherches, à paraître.

Biagioli N. (2018). Écrire à partir d'une image et fabriquer un objet à partir d'un texte : historique et caractéristiques actuelles des pratiques scolaires inter-artiales. Pratiques, à paraître.

Biagioli N. (2016). Ce qui est vivant et ce qui est mort de la didactique de l'écriture littéraire. In A. Petitjean dir. Didactiques du français et de la littérature, Recherches textuelles, (14), Université de Lorraine : CREM.

Biagioli N. (2015). Les figures au collège : un objet didactique complexe. Pratiques. L'étude des figures en contexte (163-164).

Biagioli N. (2014). Quelles relations les élèves établissent-ils entre les apprentissages langagiers extrascolaires, les apprentissages langagiers de la discipline français et ceux des autres disciplines. In B. Daunay, J.-L. Dufays dir. Didactique du français : du côté des élèves. Louvain La Neuve : de Boeck, 157-174.

Biagioli N. (2013). Biographie d'auteur imaginaire en master : entre écriture littéraire et écriture critique. In V. Houdart-Merot, Ch. Mongenot dir. Pratiques d'écriture littéraire à l'université, Paris : H. Champion, 229-242.

Biagioli [Bilous] N. (1995). L'auteur, personnage de l'histoire littéraire. Cahiers de narratologie, (6), 99-132.

Boissinot A. (2001). Expliquez les textes. In A. Boissinot, A. Armand, J. Jordy Le français en collège et en lycée. Paris : Hachette, 182-190.

Bruner J. (2002). Pourquoi nous racontons-nous des histoires? Paris : Retz.

Charaudeau P. (1998). L'argumentation n'est peut-être pas ce que l'on croit. Le Français aujourd'hui , (123), 6-15.

Condette J-F. (2006). Les recteurs d'académie en France de 1808 à 1940. T.II. Dictionnaire biographique, Histoire biographique de l'enseignement, année 2006/vol.12, (2), 102-103, consultable sur www.persee.fr/doc/inrp_0298-5632-2006_an_12_24278

Daunay B., Delcambre I. (2007). Les rituels en maternelle : genre scolaire ou disciplinaire? Les cahiers Théodile (7), 33-48.

Denizot N. (2005). L'institution scolaire des genres littéraires. Les cahiers Théodile (6), 41-62.

Eco U. [1979] (1985). Lector in fabula. Paris : Grasset. 
Falardeau E., Simard C. (2015). Le rapport à la culture : quelle culture ? La Lettre de l'AIRDF, (58), 20-24.

Giolitto P. (2003). L'Ecole paroissiale. Maîtres et écoliers de Charlemagne à Jules Ferry. Paris : Imago.

Guérin E. (2010). L'« outre-langue » des enseignants ou le mythe d'une langue monovariétale. Pratiques (en ligne) (145-146).

Halté J.-F. (1992). La didactique du français. Paris : PUF.

Houdart-Merot V. (1998). La culture littéraire au lycée depuis 1880. Rennes : PUR.

Langlade G. (2001). La lecture littéraire : théories et didactique. In A. Boissinot, A. Armand, J. Jordy Le français en collège et en lycée. Paris : Hachette, 146-151.

Marti M., Baroni R. (2014). De l'interactivité du récit au récit interactif. Cahiers de narratologie, (27).

Peyrou F. (1999). De la réécriture du maître à celle de l'élève. Le Français aujourd'hui (127), 83-90.

Plantin C. (1996). L'argumentation. Paris : Seuil.

Plantin C. (2011). Les bonnes raisons des émotions. Principes et méthodes pour l'étude du discours émotionné. Berne : Peter Lang.

Reuter Y. (2010). Forme scolaire. In Y. Reuter dir. Dictionnaire des concepts fondamentaux des didactiques. Bruxelles : De Boeck.

Veyne P. (1983). Les Grecs ont-ils cru à leurs mythes? Paris : Seuil.

\section{Manuels}

Causeret Ch. (1892) Cours élémentaire de lecture expliquée et de composition française (Livre du maître). Paris : Gedalge.

Mots et merveilles $5^{e}$. (1982). Paris : Magnard.

La littérature et les idées. (1974). Littérature et langages, t. 4. Paris : Nathan.

Rives bleues $5^{e}$. (2010). Paris : Hatier.

Terres littéraires $1^{\text {ère }}$ (2011). Paris : Hatier.

La vie en toutes lettres $1^{\text {ère }}$. (2016). Paris : Hatier.

\section{NOTES}

1. Nous différencierons désormais la discipline de la langue qu'elle enseigne par l'italique.

2. La loi Jules Ferry du 28 mars 1882 rend l'instruction obligatoire à partir de 6 ans, pour tous les enfants français ou étrangers résidant en France. À l'origine, la scolarisation était obligatoire jusqu'à l'âge de 13 ans, puis 14 ans à partir de la loi du 9 août 1936. En 1959, la réforme Berthoin repousse la fin de l'instruction obligatoire à 16 ans.

3. Afin de différencier le produit du processus de production, nous appelons « récit » le premier, et «narration » le second.

4. BO hors série $n^{\circ} 3$ du 19 juin 2008.

5. Qui englobe la $6^{\mathrm{e}}$ depuis 2015. 
6. Depuis le début du collège unique, l'apprentissage de la communication iconique est réparti entre le français pour la compréhension, et les arts plastiques pour la production (cf. Biagioli, 2017).

7. Cette progression est inchangée dans les programmes de 2008, qui prennent simplement en compte la création de la matière interdisciplinaire histoire des arts (B.O spécial $n^{\circ} 6$ du 28 août 2008).

8. Document d'accompagnement pour l'évaluation des acquis du socle commun de connaissances, de compétences et de culture, eduscol.education.fr/ressources-2016, respectivement p. 3-4,7 et 16.

9. La circulaire $\mathrm{n}^{\circ} 2010-008$ du 29 janvier 2010, BO. $\mathrm{N}^{\circ} 1$ du 4 février 2010 élargit le champ des enseignements en langue étrangère aux disciplines autres que linguistiques (DNL).

10. Projet de programme pour le cycle 4, 9 avril 2015, http://www.education.gouv.fr/cid87938/ projets-de-programmes-pour-l-ecole-elementaire-et-le-college.html, p.10 et 11 .

11. Ce n'est pas gagné si l'on se fie à un exemple assez récent. En 2009 une partie consacrée au raisonnement et à la logique a été rajoutée au programme de mathématiques de $2^{\text {de }}$. En 2014 , le rapport de la commission de suivi de la mise en œuvre des programmes de mathématiques a constaté que cet enseignement était souvent éludé, ou ne faisait l'objet que de reprises orales, et que les enseignants n'identifiaient pas la logique comme un outil de raisonnement transversal, considérant qu'elle faisait partie de l'épistémologie et des contenus de la discipline. (http:// cache.media.eduscol.education.fr/file/Mathematiques/01/6/CSM-projet-

rapport2013_293016.pdf)

12. La question de l'Homme dans les genres de l'argumentation du XVI ${ }^{\mathrm{e}}$ siècle à nos jours, présentation p. 1. MEN/DGESCO http://eduscol.education.fr/prog

\section{ABSTRACTS}

Narrative and argumentation are rival and complementary types of discourse : rivals because one can reduce the totality of the discursive experience to one as to the other, complementary because, if one chooses one of the two as the descriptive basis of language, one is obliged to resort to the other, inasmuch as both are necessary for the functioning of the enunciating triangle : 1st, 2nd, and non-person. Their teaching is therefore crucial, but also complex. Indeed, their integration into the curricula of French as a first language shows a rather spiral than a linear evolution, marked both by the variation of the theoretical frameworks of reference, their sensitivity to the socio-historical context of their teaching and the internal construction of the discipline around language-literature complementarity, which a priori subjects students' discursive practices to the objective of evaluating literary and linguistic knowledge, thus cutting them off from ordinary social practices.

The narrative is the first cultural practice in kindergarten, it is also the occasion of the first argumentative learning with the interpretative debate. Although the debate begins in kindergarten with objects other than narrative, its use as a factor of socialization marks the first stage of a didactic progression which makes oral and written argumentative competence the objective of the end of compulsory schooling (College diploma) and even higher secondary education (Advanced test of French of the French baccalauréat). For all that, the argument does not go beyond the orbit of the literary narrative, using it as well as an example, an object or a medium, while this one becomes the object of a more and more argued reading. This (con) fusion 
has led to a cultural break between school practices and the social practices of narrative and argumentation. Will the 2013 curriculum, which refocuses learning on arguments and literature, succeed in turning this vicious circle into a virtuous circle? We will answer this question after a four-parts study: sociological on the impact of academic form on narrative and discursive learning, historical on the evolution of narrative and argumentation in school practices, didactical on the curricular organization of narrative and argumentative learning, political on the redistribution of the roles of narrative and argumentation by the last school reform of 2013.

Récit et argumentation sont des types de discours à la fois rivaux et complémentaires : rivaux parce que l'on peut ramener la totalité de l'expérience discursive à l'un comme à l'autre, complémentaires parce que, si l'on choisit l'un des deux comme base descriptive du langage, on est obligé de recourir à l'autre, dans la mesure où les deux sont nécessaires au fonctionnement $\mathrm{du}$ triangle énonciatif: $1^{\text {ère }}, 2^{\text {ème }}$ personnes et non -personne. Leur didactisation est donc cruciale, mais également complexe. En effet, leur intégration dans les curriculums du français langue première, montre une évolution plutôt spiralaire que linéaire, marquée à la fois par la variation des cadres théoriques de référence, leur sensibilité au contexte socio-historique de leur enseignement et la construction interne de la discipline autour de la complémentarité languelittérature, qui soumet a priori les pratiques discursives des élèves à l'objectif d'évaluation des savoirs littéraires et langagiers, les coupant ainsi des pratiques sociales ordinaires.

Le récit est la première pratique culturelle scolarisée à la maternelle, c'est aussi l'occasion du premier apprentissage argumentatif avec le débat interprétatif. Bien que le débat s'ouvre dès la maternelle à d'autres objets que le récit, son utilisation comme facteur de socialisation marque le premier stade d'une progression didactique qui fait de la compétence argumentative orale et écrite l'objectif de la fin de la scolarité obligatoire (Brevet des Collèges) et plus encore des études secondaires supérieures (Épreuve anticipée du baccalauréat de français). Pour autant, l'argumentation ne sort guère de l'orbite du récit littéraire, qu'il lui serve d'exemple, d'objet ou de support, tandis que celui-ci fait l'objet d'une lecture de plus en plus argumentée. Cette (con)fusion a entraîné une coupure culturelle entre les pratiques scolaires et les pratiques sociales du récit et de l'argumentation. Les programmes de 2013, qui recentrent les apprentissages autour de l'argumentation et de la littérature, parviennent-ils à transformer ce cercle vicieux en cercle vertueux ? Nous répondrons à cette question à l'issue d'une étude en 4 parties: sociologique sur l'impact de la forme scolaire sur les apprentissages narratifs et discursifs, historique sur l'évolution du récit et de l'argumentation dans les pratiques scolaires, didactique sur l'organisation curriculaire des apprentissages narratifs et argumentatifs, politologique sur la redistribution des rôles du récit et de l'argumentation par la dernière réforme scolaire de 2013.

\section{INDEX}

Mots-clés: récit, argumentation, forme scolaire, français langue première, configuration disciplinaire, progression didactique, politique éducative

Keywords: narrative, argumentation, academic form, French first language, disciplinary configuration, didactical progression, school policy 


\section{AUTHOR}

\section{NICOLE BIAGIOLI}

ESPE - I3DL (Université Côte d'Azur), 89 Avenue George V, 06046 Nice cedex 1, biagioli@unice.fr Nicole Biagioli est narratologue, didacticienne du français, et dirige le laboratoire I3DL.

Interdidactique, Didactique des disciplines et des langues, UNS. Elle s'intéresse à la transposition didactique des notions qui permettent de penser les relations entre récit et argumentation à l'oral et à l'écrit dans les différentes disciplines, à l'école et à l'université.

\section{Références bibliographiques :}

-(2016) Biagioli N. Ce qui est vivant et ce qui est mort de la didactique de l'écriture littéraire. A. Petitjean dir. Didactiques du français et de la littérature, Recherches textuelles, $\mathrm{n}^{\circ} 14$, Université de Lorraine, publications du CREM.

-(2015) Biagioli N. Les figures au collège : un objet didactique complexe, Pratiques, L'étude des figures en contexte (G. Salvan, L. Gaudin-Bordes coord), 163-164.

-(2014) Biagioli N. Quelles relations les élèves établissent-ils entre les apprentissages langagiers extrascolaires, les apprentissages langagiers de la discipline français et ceux des autres disciplines. B. Daunay, J.-L. Dufays dir. Didactique du français : du côté des élèves. Louvain La Neuve: DeBoeck, 157-174.

-(2013) Biagioli N. Biographie d'auteur imaginaire en master : entre écriture littéraire et écriture critique. V. Houdart-Merot, Ch. Mongenot dir. Pratiques d'écriture littéraire à l'université, Paris :H.

Champion, 229-242. 